\title{
Classical capillary turbulence on the surface of quantum liquid $\mathrm{He}-\mathrm{II}$
}

\author{
L.V. Abdurakhimov, M.Yu. Brazhnikov, I.A. Remizov, and A.A. Levchenko \\ Institute of Solid State Physics RAS, Chernogolovka, Moscow district. 142432, Russia \\ E-mail: abdurl@issp.ac.ru
}

Received June 14, 2010

\begin{abstract}
Superfluid helium-4 is a unique liquid for experimental study of capillary wave turbulence due to its very low viscosity. We have studied the influence of amplitude and spectral characteristics of the excitation force on the behavior of the turbulent cascade of capillary waves in the cylindrical cell of diameter $30 \mathrm{~mm}$. We have found that the experimental results can be explained within the framework of the wave turbulence theory (WTT) when the amplitude of pumping is relatively high. However, a very interesting phenomenon was observed at moderate amplitudes of harmonic surface excitation. It was detected the deviation of the turbulent spectrum from the power law function predicted by WTT at high frequencies — local maximum - that can be interpreted as wave energy accumulation. Our estimations show that the special case of wave turbulence is realized in our measurements, namely, discrete turbulence which is characterized by the strong influence of discreteness of cell resonant frequencies on the mechanism of the nonlinear interaction.

PACS: $68.03 . \mathrm{Kn}$ Dynamics (capillary waves);

47.27.Gs Isotropic turbulence; homogeneous turbulence.
\end{abstract}

Keywords: capillary turbulence, discrete turbulence, superfluid helium-4.

\section{Introduction}

Wave turbulence (WT) is a non-equilibrium state of the weakly nonlinear wave system wherein scales of energy pumping and energy dissipation are well-separated [1]. Examples of this nonlinear phenomenon can be found in diverse fields such as plasma physics, nonlinear optics, ferromagnetism, and hydrodynamics. In particular, an ensemble of nonlinear capillary waves on the liquid surface is a well-known system in which wave turbulence can be realized. For capillary waves the main processes of the nonlinear interaction are three-wave processes of decay and merging that satisfy the conservation laws of frequency $\omega$ and wave-vector $\mathbf{k}$ : $\omega_{1}+\omega_{2}=\omega_{3}, \quad \mathbf{k}_{1}+\mathbf{k}_{2}=\mathbf{k}_{3}$. The WT theory predicts that a direct turbulent cascade is formed in the inertial range between the energy pumping scale and energy dissipation scale with the wave spectrum described by the power law function of frequency (Kolmogorov-Zakharov spectrum). In recent years, due to the development of technical equipment and computational tools for data processing, capillary turbulence has been in focus of experimental studies of capillary waves on the surface of water [2-4], ethanol [4], silicon oil [5] and mercury [6]. Our previous experiments [7,8] showed that the use of quantum fluids (liquid ${ }^{4} \mathrm{He}$ and liquid hydrogen) for investigations of capillary turbulence can provide additional advantages with respect to traditional liquids due to their low viscosity. In the present paper we focus on the peculiarities of the shape of the turbulent wave energy distribution at high frequencies observed in experiments with He II, a superfluid phase of liquid ${ }^{4} \mathrm{He}$ (preliminary results were published in [9]). For the first time we observed that under certain conditions a deviation from the power law spectrum is formed at frequencies of the order of the viscous cut-off scale. We also suggest a qualitative model of this phenomenon that takes into account the influence of discreteness arising due to the finite sizes of the experimental cell.

\section{Experimental set-up}

In the reported experiments with superfluid ${ }^{4} \mathrm{He}$ we used the technique similar to that of our previous studies of capillary turbulence on the surface of liquid hydrogen [10]. The measurements were made at temperature $T \approx 1.7 \mathrm{~K}$ (for comparison, the temperature of the normal-tosuperfluid state transition for liquid ${ }^{4} \mathrm{He}$ at saturated vapor pressure is $T_{\lambda}=2.17 \mathrm{~K}$ ). At this temperature superfluid helium has kinematic viscosity $v=8.9 \cdot 10^{-5} \mathrm{~cm}^{2} / \mathrm{s}$, density $\rho=0.145 \mathrm{~g} / \mathrm{cm}^{3}$, surface tension $\sigma=0.32 \mathrm{din} / \mathrm{cm}[11]$. Liquid helium was condensed into a copper cup of inner diameter $D=30 \mathrm{~mm}$ and depth $4 \mathrm{~mm}$. At the bottom of 
the cup a radioactive thin plate was placed. Emitted from the plate $\beta$-electrons ionized ${ }^{4} \mathrm{He}$ atoms which led to formation of positively charged ions and electrons. Between the cup and the upper electrode (a copper plate placed in vapor at a distance of $3 \mathrm{~mm}$ above the liquid surface) constant dc voltage $U \approx 600 \mathrm{~V}$ of appropriate polarity was applied, so that the liquid surface was charged by positive ions. By applying low-frequency ac voltage with amplitudes from 1 up to $20 \mathrm{~V}$ to the cup, in addition to de voltage, the capillary waves were excited on the charged surface. The advantage of this method of wave pumping is that the surface is excited directly by the driving force, rather than instability as in the experiments with parametrically excited waves $[2,4,5]$ and not by blade-wavemakers as in [6] which probably resulted in any motion in the bulk. Oscillations of the surface were detected by measurements of the power of the laser beam reflected from the surface. The laser beam fell on the surface at sliding angle $\alpha \approx 0.15$ producing an elliptic spot of size $1 \mathrm{~mm} \times 7 \mathrm{~mm}$. Typical wavelengths of capillary waves in the experiments were smaller than the spot dimensions. The laser beam reflected from the oscillating surface was focused by the lens on a Si photodiode (Hamamatsu s3590-08). Time variations of the signal from the photodiode were amplified by a SR570 amplifier and then digitized by a 24-bit analogueto-digital converter Leonardo-II [12] with discretization frequency $102.4 \mathrm{kHz}$. Amplified signal from the diode was proportional to the time variations of the reflected laser beam power $P(t)$. Signal $P(t)$ was recorded to the hard disk into a series of 16 files of approximately 5 seconds long each. From Fast Fourier Transform (FFT) of every data file, power spectrum $\left|P_{\omega}\right|^{2}$ was computed, and averaging was performed over all the 16 files. As it was shown in our previous works (see, for example, $[8,10]$ ) the reflected laser beam power spectrum $\left|P_{\omega}\right|^{2}$ was proportional to the spectrum of the correlation function of surface elevation $I_{\omega}=\left|\eta_{\omega}\right|^{2}$, hence $\left|P_{\omega}\right|^{2} \sim\left|\eta_{\omega}\right|^{2}$.

In the reported measurements two types of lowfrequency driving force were used. In the first type experiments the surface was driven by a sinusoidal force with frequency $\omega_{\text {pump }}$ that was equal to that of resonant frequencies of the cell. In the second type measurements driving by a noise force was used. In this case the electrical noise was produced by a programmable noise generator.

\section{Results}

Figure 1 shows the turbulent spectrum of capillary waves $P_{\omega}^{2}$ obtained in our experiments when the surface was pumped by ac voltage at frequency $80 \mathrm{~Hz}$ and amplitude $11 \mathrm{~V}$. Turbulent distribution $P_{\omega}^{2}$ looks similar to that observed earlier in our experiments on the surface of liquid hydrogen [7] and in our first experiments on the surface of liquid ${ }^{4} \mathrm{He}[8]$. The spectrum consists of equidistant harmonics: the first harmonic frequency corresponds to the

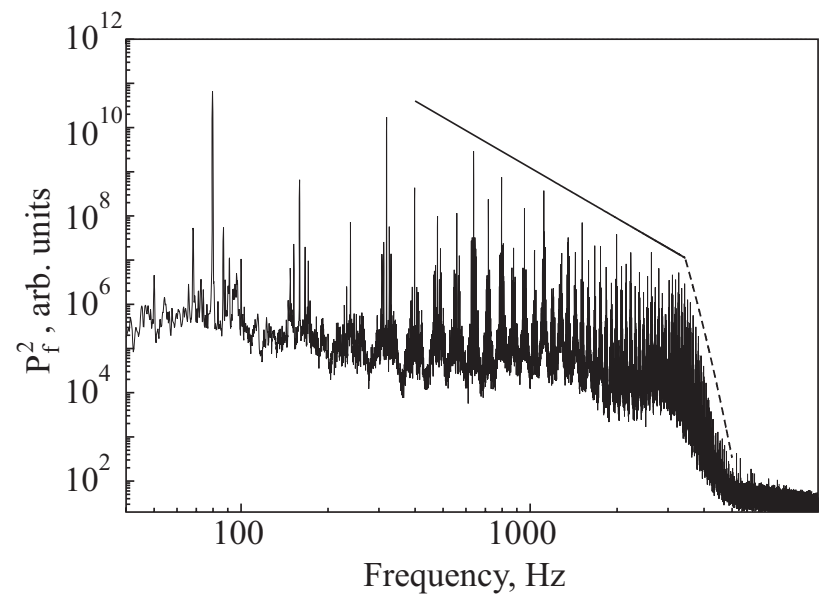

Fig. 1. Turbulent spectrum of capillary waves on He II surface ( $T=1.7 \mathrm{~K}$ ) obtained when the surface was pumped by sinusoidal force with frequency $80 \mathrm{~Hz}$, amplitude of pumping voltage $11 \mathrm{~V}$. Straight line corresponds to power law $P_{\omega}^{2} \sim \omega^{-3.7}$ predicted by the theory for narrow-band pumping. Dashed curve corresponds to $\sim \exp (-f / 150)$.

pumping frequency, while other harmonics are generated due to the nonlinear interactions at frequencies multiple to the pumping frequency. In the inertial range the amplitudes of the harmonics depend on frequency as power law function $P_{\omega}^{2} \sim \omega^{-m}$ with index $m \approx 3.7$ which is in accordance with the theoretical prediction for narrow-band pumping [13]. At frequencies of about $4 \mathrm{kHz}$ the wave amplitudes were reduced significantly as a result of viscous dissipation and tended to the level of instrumental noise.

Similarly, when the surface was forced by an intense noise pumping in the frequency range $90-200 \mathrm{~Hz}$, power law spectrum $P_{\omega}^{2} \sim \omega^{-2.8}$ was obtained (Fig. 2). Index $\sim 2.8$ is predicted by the theory for broad-band pumping [1], and the difference of the indices in the experiments with har-

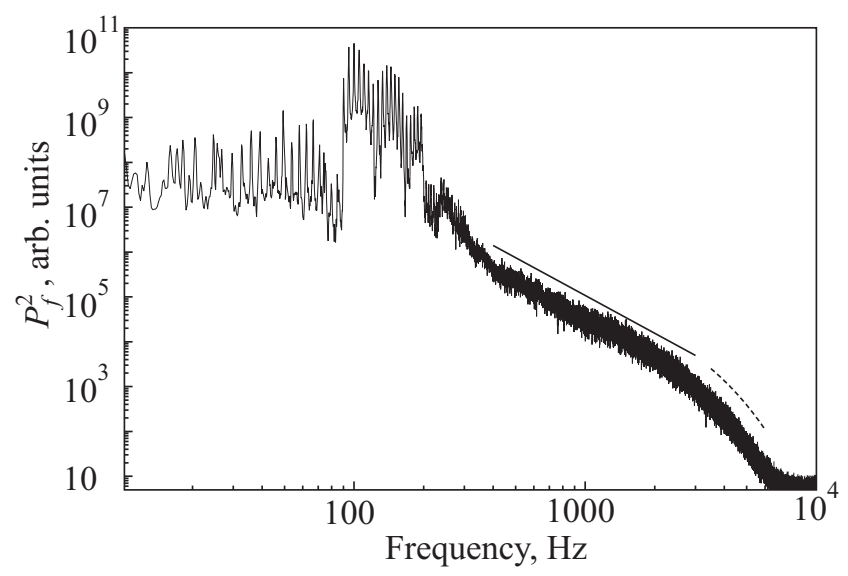

Fig. 2. Turbulent spectrum of capillary waves in the case of noise pumping at 90-200 Hz. Straight line corresponds to power law $P_{\omega}^{2} \sim \omega^{-2.8}$ predicted by the theory for broad-band pumping. Dashed curve corresponds to $\sim \exp (-f / 800)$. 
monic pumping and broad-band noise pumping is in accordance with our previous experiments on liquid hydrogen [14]. It is interesting to note, that, within a dissipative frequency range, the wave amplitudes in the turbulent spectrum generated by a harmonic force are decreased more rapidly than those in the spectrum generated by a noise force (exponential dashed curves in Figs 1,2).

When the amplitude of harmonic pumping was decreased to $10 \mathrm{~V}$, the shape of the turbulent spectrum is changed (Fig. 3). High-frequency edge of the turbulent cascade is shifted towards low frequencies which is in accordance with our previous experiments $[8,15]$. However a new phenomenon was observed, namely, formation of a well-pronounced local maximum in the wave amplitudes at frequencies of the order of high-frequency edge of turbulent cascade $\omega_{b}$ (shown by the dashed curve in Fig. 3). With further reduction of the pumping amplitude, the local maximum was shifted towards low frequencies. Eventually, at very low pumping amplitudes the spectrum was consisted only of a few harmonics, and no local maximum was found.

Formation of a local maximum was seen in a number of experiments with harmonic pumping. The common feature observed in the experiments was that the local maximum was formed near the high-frequency edge of the turbulent cascade although the shape of the local maximum and its exact position depended on the pumping frequency as well as on the pumping amplitude. For example, when the surface was pumped by the sinusoidal force at $34 \mathrm{~Hz}$, the wave spectrum with a distinct local maximum was obtained (Fig. 4). However, the local maximum was located in the dissipation region of turbulent distribution rather than in the inertial range as is shown in Fig. 3.

In the case of noise pumping, we never observed a local maximum. To illustrate this, in Fig. 5 we present a turbulent distribution obtained when the surface was pumped by a

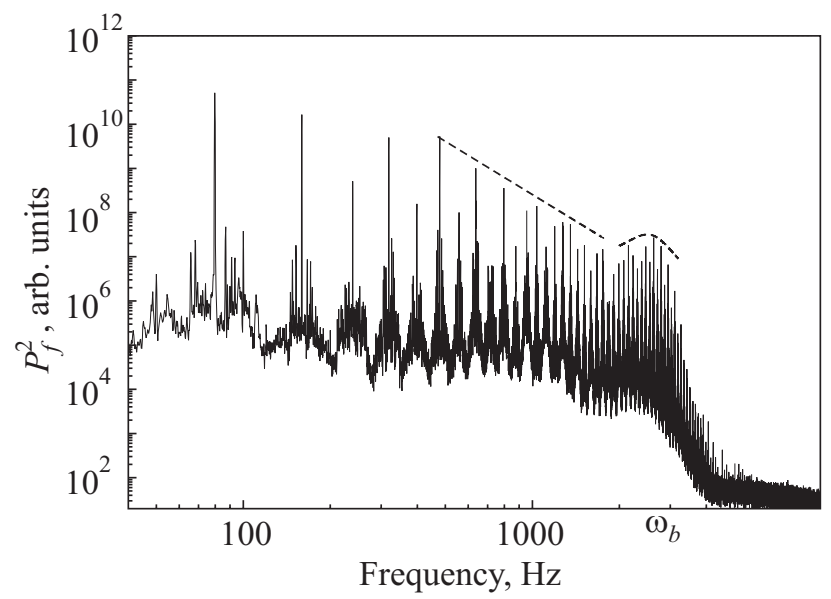

Fig. 3. Turbulent spectrum after reduction of pumping amplitude. Frequency of pumping $80 \mathrm{~Hz}$, amplitude of pumping voltage $10 \mathrm{~V}$. At frequencies of about $2.5 \mathrm{kHz}$, a local maximum is observed (emphasized by dashed curve). Dashed straight line corresponds to $P_{\omega}^{2} \sim \omega^{-3.7}$.

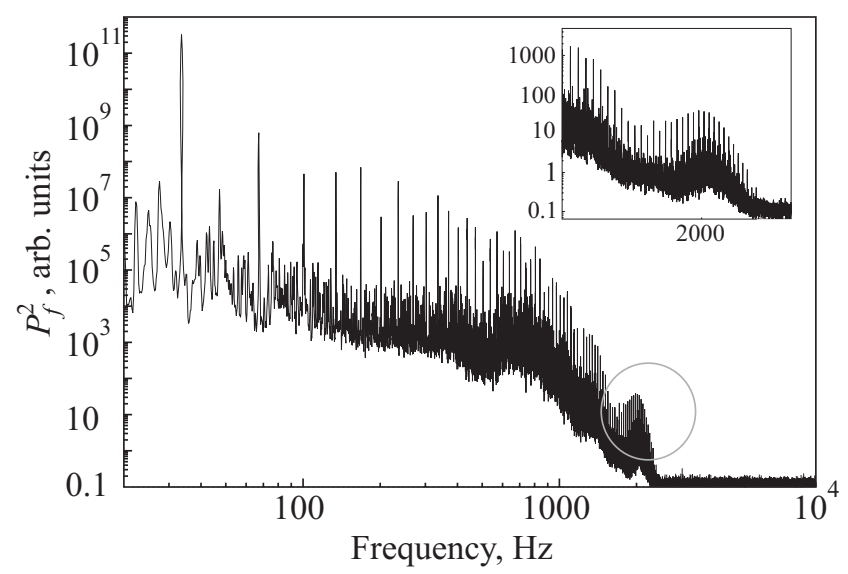

Fig. 4. Turbulent spectrum in the case of pumping at $\omega_{\text {pump }} / 2 \pi=34 \mathrm{~Hz}$. Formation of a maximum at the spectrum is clearly seen on the main plot (circled) and in the insert on an enlarged scale.

noise signal in the frequency range $60-130 \mathrm{~Hz}$. The amplitude of pumping force was chosen so that the highfrequency edge of inertial interval was located approximately at those frequencies at which it was seen in Figs. 3, 4. The spectrum differs drastically from the spectra observed in the experiments with harmonic pumping: it is continuous and the spectrum shape at high frequencies is more smooth.

\section{Discussion}

Formation of the local maximum can be interpreted as the wave energy accumulation at the high-frequency edge of the turbulent cascade where transition from the nonlinear wave energy transfer to viscous damping occurs. In the theoretical work [16] it is shown that viscous dissipation (which leads to finiteness of the inertial range) can in principle result in the energy accumulation due to the bottleneck effect. However, it is not clear in which manner the dependence of formation of the local maximum on the

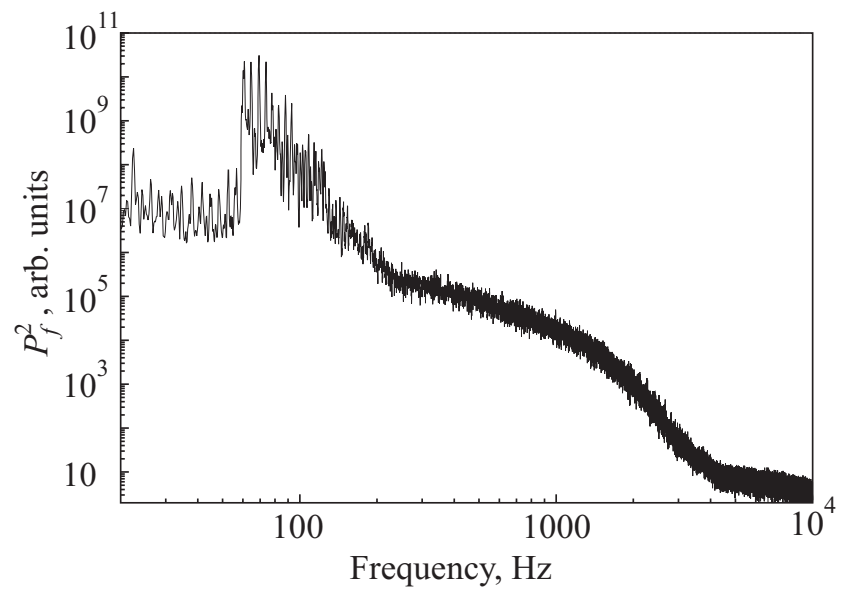

Fig. 5. Turbulent spectrum of capillary waves in the case of noise pumping at $60-130 \mathrm{~Hz}$. 
pumping amplitude observed in our measurements can be explained by the influence of viscous dissipation. On the other hand, it was pointed out by E. A. Kartashova [17,18] that there were peculiarities in weakly nonlinear wave interactions in resonators due to discreteness of wavenumber space. Theory of discrete wave turbulence was developed later in a number of works, but of particular interest for us is the work [19] where the model of "frozen" turbulence was proposed. It follows from this model that, at small amplitudes of pumping, discreteness of wavenumber space can result in an oscillatory wave spectrum. We suppose that the local maximum is also caused by the discreteness of $k$-space. However, the above-mentioned work [19] considers the case of waves on the surface with square boundary, when the wavenumber $k$-space is two-dimensional. Otherwise, in our experiments the boundary geometry is cylindrical, surface oscillations should be described by the Bessel functions, and the wavenumber space is onedimensional. It is known that the form of the basin boundary strongly affects the density of resonant wavenumbers [18]. Therefore, we performed the following alternative estimations to understand the influence of discreteness on spectrum in our experiments. We suppose that the phenomenon is caused by the detuning of frequencies of the harmonics (shown in Figs. 1, 3, 4) and the resonant frequencies of the cell. For surface waves in a cylindrical resonator with diameter $D$ the eigenvalues of wavenumber $k$ are the roots of boundary condition equation $J_{1}(k D / 2)=0$, where $J_{1}(x)$ is a Bessel function of the first order. When $k$ is large, the asymptotic form of the Bessel function can be used, and the resonance wavenumbers are equidistant with step $\Delta k \approx 2 \pi / D$. Hence, the distance between two neighbour resonance frequencies should grow with increasing frequency as

$$
\Delta \omega=\frac{\partial \omega}{\partial k} \Delta k \approx \frac{\partial \omega}{\partial k} \frac{2 \pi}{D}=\frac{3 \pi}{D}\left(\frac{\sigma}{\rho}\right)^{1 / 3} \omega^{1 / 3} .
$$

Thus, for capillary waves the resonant frequencies are not equidistant which is in contrast to the frequencies of harmonics that are multiple to the pumping frequency $\omega_{\text {pump }}$ (it should be mentioned, that in our experiments $\left.\Delta \omega<\omega_{\text {pump }}\right)$.

Obviously, such detuning is important at frequencies where broadening of resonance $\delta \omega$ is smaller than the distance between two neighbour resonances $\Delta \omega$ (see Fig. 6)

$$
\frac{\delta \omega}{\Delta \omega}<1
$$

Resonance broadening $\delta \omega$ can be presented as a sum of viscous broadening $\delta \omega_{v}$ and nonlinear broadening $\delta \omega_{n l}$,

$$
\delta \omega=\delta \omega_{v}+\delta \omega_{n l}
$$

The viscous resonance broadening corresponds to the viscous damping [20]

$$
\delta \omega_{v}=4 v k_{\omega}^{2}=4 v\left(\frac{\rho}{\sigma}\right)^{2 / 3} \omega^{4 / 3},
$$

and is related to characteristic time of viscous dissipation $\tau_{v}$ as $\delta \omega_{v}=\tau_{v}^{-1}$. Nonlinear resonance broadening corresponds to nonlinear transfer of wave energy and is determined from characteristic time of nonlinear interaction $\tau_{n l}$ as $\delta \omega_{n l}=\tau_{n l}^{-1}$. We suppose that for the case of harmonic pumping nonlinear time $\tau_{n l} \sim \omega^{1 / 6}$ [21] and consequently $\delta \omega_{n l} \sim \omega^{-1 / 6}$. With increase of the amplitude of pumping $A$ the nonlinearity should increase too, therefore it can be assumed that

$$
\delta \omega_{n l} \approx \varepsilon(A) \omega^{-1 / 6},
$$

where $\varepsilon(A)$ is the increasing function of driving amplitude $A$.

Thus, by substituting (1),(3),(4),(5) in (2), we obtain that detuning is important at frequencies where

$$
\frac{4 v(\rho / \sigma)^{2 / 3} \omega^{4 / 3}+\varepsilon(A) \omega^{-1 / 6}}{(3 \pi / D)(\sigma / \rho)^{1 / 3} \omega^{1 / 3}}<1 .
$$

At the high-frequency edge of inertial range $\omega_{b}$, where damping of the turbulent cascade is observed, the nonlinear wave energy transfer is changed by viscous dissipation. Therefore, it can be assumed that the characteristic viscous time is close to the characteristic nonlinear time at $\omega_{b}$, $\tau_{v}\left(\omega_{b}\right) \approx \tau_{n l}\left(\omega_{b}\right)$. Thus, although function $\varepsilon(A)$ is unknown, it can be estimated from the condition that at the edge frequency $\omega_{b}$ the nonlinear broadening $\delta \omega_{n l}=\tau_{n l}^{-1}$ and the viscous broadening $\delta \omega_{v}=\tau_{v}^{-1}$ are equal,

$$
\delta \omega_{v}\left(\omega_{b}\right) \approx \delta \omega_{n l}\left(\omega_{b}\right) .
$$

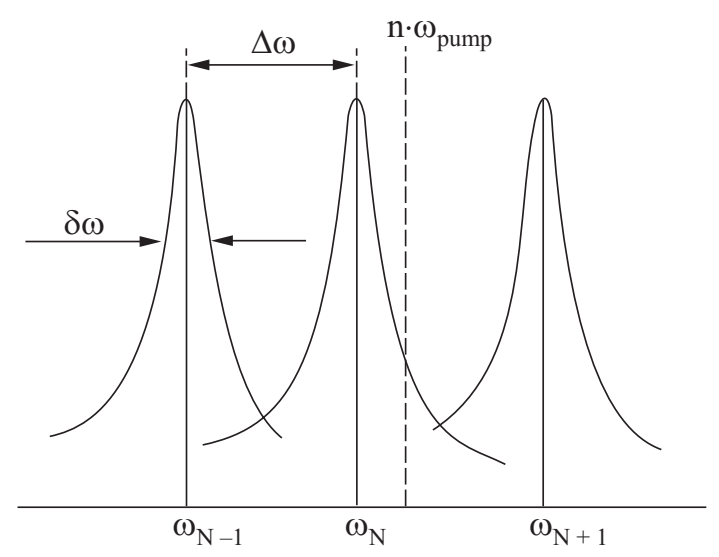

Fig. 6. When broadening of resonance $\delta \omega$ is smaller than distance between two neighbour resonances $\Delta \omega$, some harmonics are out of the resonances of surface oscillations in the experimental cell. 
It follows from our estimations that, in our experiments, condition (6) is satisfied in the frequency domain near cutoff viscous frequency $\omega_{b}$ (both below and above $\omega_{b}$, see Fig. 7). In this frequency domain the detuning of the harmonic frequencies and of the resonant frequencies becomes essential, and it determines the specific shape of turbulent distribution $P_{\omega}^{2}$. However, based upon a simple qualitative reasoning it is impossible to predict the exact shape and position of the local maximum. Thus, detailed theoretical studies and numerical simulations are required to understand the mechanism of formation of the local maximum.

The model proposed implies that the following conditions should be satisfied for observation of energy accumulation in the case of capillary turbulence: low viscous broadening (low viscosity of liquid), low nonlinear broadening (harmonic pumping with a moderate amplitude), and large distance between the resonant frequencies (a relatively small size of the cell). In our experiments these conditions were fulfilled. It is interesting to note that the use of superfluid ${ }^{4} \mathrm{He}$ allowed us to observe the energy accumulation in turbulent cascade due to its very low viscosity in comparison with classical liquids.

We are grateful to L.P. Mezhov-Deglin, G.V. Kolmakov, E.A. Kuznetsov for fruitful discussions and A.V. Lokhov for experimental assistance. The work was supported by RFBR grants 09-02-01146, 07-02-00728 and the programs of the Presidium RAS "Quantum Physics of Condensed Matter" and "Fundamental Problems of Nonlinear Dynamics".

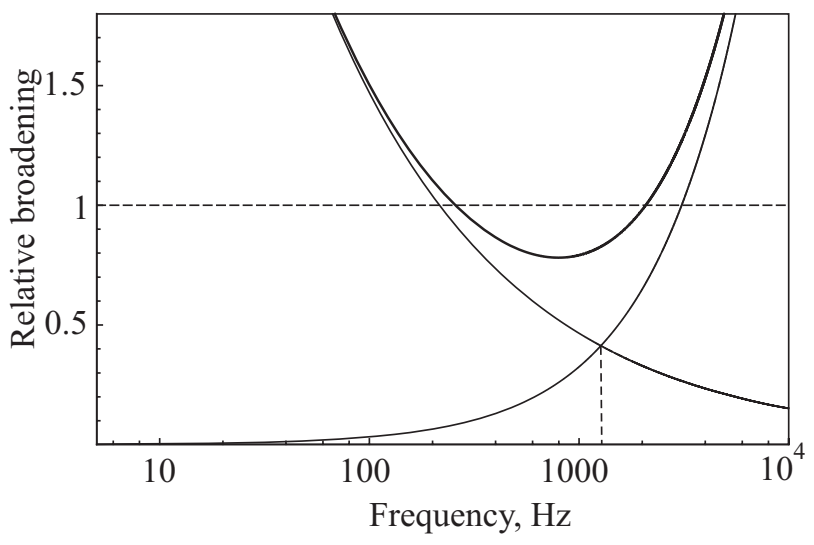

Fig. 7. Results of estimation of relative broadening $\delta \omega / \Delta \omega$. Ascending thin curve represents viscous relative broadening $\delta \omega_{v} / \Delta \omega$, descending thin curve - nonlinear relative broadening $\delta \omega_{n l} / \Delta \omega$, thick curve with minimum - total relative broadening $\delta \omega / \Delta \omega$.
1. V. Zakharov, V. Lvov, and G. Falkovich, Kolmogorov Spectra of Turbulence, Vol. 1, Springer, Berlin (1992).

2. W.B. Wright, R. Budakian, S.J. Putterman, Phys. Rev. Lett. 76, 4528 (1996); E. Henry, P. Alstrom, and M.T. Levinsen, Europhys. Lett. 52, 27 (2000); H. Punzmann, M.G. Shats, and H. Xia, Phys. Rev. Lett. 103, 064502 (2009).

3. M.Yu. Brazhnikov, G.V. Kolmakov, A.A. Levchenko and L.P. Mezhov-Deglin, Europhys. Lett. 58, 510 (2002).

4. C. Falcon, E. Falcon, U. Bortolozzo, and S. Fauve, Europhys. Lett. 86, 14002 (2009).

5. Mark-Tiele Westra, Patterns and Weak Turbulence in Surface Waves, ISBN 90-386-1749-6, Eindhoven (2001).

6. E. Falcon, C. Laroche, and S. Fauve, Phys. Rev. Lett. 98, 094503 (2007).

7. L.V. Abdurakhimov, M.Yu. Brazhnikov, and A.A. Levchenko, JETP Lett. 89, 120 (2009); L.V. Abdurakhimov, M.Yu. Brazhnikov, and A.A. Levchenko, JETP Lett. 88, 19 (2008); G.V. Kolmakov, M.Y. Brazhnikov, A.A. Levchenko, L.V. Abdurakhimov, P.V.E. McClintock, and L.P. MezhovDeglin, in: Progress in Low Temperature Physics, Quantum Turbulence, Elsevier, Oxford (2009), Vol. XVI, p. 306.

8. L.V. Abdurakhimov, M.Yu. Brazhnikov, and A.A. Levchenko, Fiz. Nizk. Temp. 35, 127 (2009) [Low Temp. Phys. 35, 95 (2009)].

9. L.V. Abdurakhimov, M.Yu. Brazhnikov, G.V. Kolmakov, and A.A. Levchenko, J. Phys.: Conf. Series 150, 032001 (2009).

10. M.Yu. Brazhnikov, A.A. Levchenko, and L.P. MezhovDeglin, Instruments Exp. Techn. 45, 758 (2002).

11. R.J. Donnelly and C.F. Barenghi, J. Phys. Chem. Ref. Data 27, 1217, URL http://darkwing.uoregon.edu/ rjd/vapor1.htm (1998).

12. http://www.rudshel.ru/pdf/Leonardo-II.rar

13. G.E. Falkovich and A.V. Shafarenko, Sov. Phys. JETP 67, 1393 (1988).

14. M.Yu. Brazhnikov, G.V. Kolmakov, and A.A. Levchenko, JETP 122, 521 (2002).

15. M.Yu. Brazhnikov, G.V. Kolmakov, A.A. Levchenko, and L.P. Mezhov-Deglin, JETP Lett. 74, 583 (2001).

16. I.V. Ryzhenkova and G.E. Falkovich, Sov. Phys. JETP 71, 1085 (1990).

17. E.A. Kartashova, Physica D46, 43 (1990).

18. E.A. Kartashova, Physica D54, 125 (1991).

19. A.N. Pushkarev and V.E. Zakharov, Physica D135, 98 (2000).

20. L.D. Landau and E.M. Lifshitz, Fluid Mechanics, Course of Theoretical Physics, Pergamon Press (1987) Vol. 6.

21. G.V. Kolmakov, JETP Lett. 83 (2), 58 (2006). 